\title{
Large Retrospective Study of Traumatic Brain Injury undergo De compressive Craniotomy
}

\author{
Rhys Payne ${ }^{1 *}$, and Aaron Rudi ${ }^{1}$.
}

${ }^{1}$ Department of Neurosurgery, Russia.

* Corresponding Author: Rhys Payne, Department of Neurological, Russia.

Received date: February 25, 2019; Accepted date: March 20, 2019; Published date: March 28, 2019.

Citation: Rhys Payne, and Aaron Rudi. Large Retrospective Study of Traumatic Brain Injury undergo De compressive Craniotomy. Brain and Neurological Disorders. 2(1); Doi: 10.31579/2642-973X/009

Copyright: @ 2019 Rhys Payne. This is an open-access article distributed under the terms of The Creative Commons Attribution License, which permits unrestricted use, distribution, and reproduction in any medium, provided the original author and source are credited.

\begin{abstract}
Decompressive craniectomy is widely used for treating patients with traumatic brain injury (TBI). Usually patients have dura mater defect due to surgery or injury itself. The defective area may left open or repaired by artificial dura substitutes. A variety of artificial dura substitutes have been used for this purpose.
\end{abstract}

Keywords: artificial dura material, bovine-derived pericardium decompressive craniectomy, traumatic brain injury.

\section{Introduction}

Traumatic brain injury (TBI) is a complex injury with a broad spectrum of symptoms and disabilities, and it is the leading cause of death for individuals in our society between the ages of 1 and 45 (Rutland-Brown, Langlois, Thomas, \& Xi, 2006). Many survivors live with significant disabilities, resulting in major socioeconomic burden (Rutland-Brown et al., 2006). Most of patients have mixed brain injuries, including brain concussion, subdural hematoma, and subarachnoid hemorrhage (Saatman et al., 2008). Patients with severe brain injury, based upon neurologic status defined by the Glasgow coma scale (GCS), usually need emergent surgical intervention, and decompressive craniectomy is a common procedure to release intracranial pressure (ICP) and open up brain cavity for further surgical treatment (Compagnone et al., 2005). Many patients may have significant dura mater defect due to TBI itself or by surgery. The defective dura area may be left open without repair or sealed with artificial dura substitutes (Huang, Lee, Chen, \& Wang, 2011; Mundinger et al., 2016; Wang et al., 2015; Zhang, Yang, Jiang, \& Zeng, 2010). Both procedures have been widely used and have distinctive advantages and disadvantages, for instance, patients without dura repair may have increased risk of CSF leakage and infection, while have better decompressive effect. In the meantime, dura repair with ADM may cause foreign body reaction and tissue capsule formation; however, it prevents CSF leakage and reduces the risk of intracranial infection (Cho \& Kang, 2017; Zhang et al., 2017). So far there lacks of large clinical studies to compare clinical outcomes of both procedures in TBI patients.

Many materials have been studied for dura mater repair in patients with TBI, including silicon-coated Dacron (Ongkiko, Keller, Mayfield, \& Dunsker, 1984), reconstituted collagen foil (Pettorini et al., 2010), expanded polytetrafluoroethylene (ePTFE) porous material (Matsumoto et al., 2013), bioabsorbable polymers (Yamada et al., 2002), and nonabsorbable Neuro-Patch (Huang et al., 2011). And some newly developed biomaterials show promising effect in animal models, such as poly (glycolide-co-lactide)/type I collagen/chitosan artificial (Bai, Wang, Yuan, Wang, \& Wang, 2013), and cellulose knitted fabric (Suwanprateeb et al., 2016). Complications, such as capsule formation, hemorrhage, and extra-axial hematoma formation, were reported caused by these materials (Huang et al., 2011; Matsumoto et al., 2013; Ongkiko et al., 1984). Recently, equinederived pericardium membrane was used a novel material for dura repair and showed significant therapeutic effect (Centonze, Agostini, Massaccesi, Toninelli, \& Morabito, 2016).
In this pilot study, none of the eight patients exhibited CSF leak, cerebral contusion, hemorrhage, or wound infection, as well as pseudomeningocele in a follow-up examine (Centonze et al., 2016), indicating xenograft membrane is a good source for artificial dura substitute.

\section{Patients And Methods}

Criteria for patient selection: Totally 387 patients who had traumatic craniectomy from January 2011 to December 2013 in Affiliated Hospital of Logistics University of People's Armed Police Force were included in this study. A total of 192 patients from January 2011 to June 2012 in our hospital were treated with standard decompressive craniectomy without dura repair. After July 2012, dura mater repair was recommended for TBI patients as guideline changed, and 195 patients who received dura repair from July 2012 to December 2013 were included in this study. All patients had severe TBI based on the Glasgow coma scale (GCS $\leq 8)$. Most of them have complicated trauma, including subdural hematoma, brain contusion, intracranial hematoma, and subarachnoid hemorrhage.

\section{Artificial dura substitutes}

Biological dura mater patches were manufactured by Guanhao Biotech (Guangzhou, China). Briefly, bovine pericardium was harvested from 12month-old 400-500 kg bovines (Chongqing Hengdu Food Development Co., Ltd. China) within $4 \mathrm{hr}$ of sacrifice. The pericardium membranes were decellularized based on a previous study (Freytes, Martin, Velankar, Lee, \& Badylak, 2008). The pericardium membranes were placed in aqueous peracetic acid $(0.1 \% \mathrm{v} / \mathrm{v})$ /ethanol $(4 \% \mathrm{v} / \mathrm{v})$ solutions for $2 \mathrm{hr}$. In the next step, pericardium membranes were washed by phosphate-buffered saline (PBS, pH 7.4) and sterile deionized water, so as to remove the peracetic acid residue thoroughly. The decellularized pericardium membranes were fixed, and biological dura mater patches were fabricated. The biological dura mater patch was packaged and sterilized by $\gamma$ irradiation at $25 \mathrm{kGy}$ using a 60Co source. The dura patches were tested negative with prion protein as well as other pathogens, and it has met the criteria of China Food and Drug Administration regulation.

\section{Surgical procedures}

All the patients were under general anesthesia with airway intubation. The blood pressure, heart rate, and respiratory rate were closely monitored by anesthesiologists, and standard decompressive craniectomies were performed. The dura was cut in radial pattern or in arch shape, and damaged brain tissues and blood clot were removed. In ADM group, the dura was suspended along the cranial window, and defective areas were fixed by decellularized bovine-derived pericardium membranes. 
The artificial membrane was decompressive sutured and sealed to the remaining dura mater. In control group, the dura was suspended along the cranial window, and the defective areas were left open without repair. In both groups, postoperative complications were closely monitored, including subcutaneous hematoma, foreign body reaction, intracranial infection, seizure, and fever. Information of surgery, such as duration of the operation, and blood loss, as well as degree of dura damage, was also collected for all patients.

\section{Results}

\section{Demographic information of Patients}

Totally 387 patients with severe TBI were included in this study, 192 patients were treated with standard decompressive craniectomy without dura repair (Control group), and 195 patients were treated with decompressive craniectomy followed artificial dura material repair (ADM group). A total of 150 male and 45 female patients were included in ADM group with average age of $43.83 \pm 15.13$ years old, and control group consisted of 130 male and 62 female patients with average age of $41.74 \pm 15.67$ years old (Table 1). There is no age and sex difference in both groups (Table 1). Patients from both groups have comparable severity of TBI based on the GCS scales obtained on initial examination (7.457.45 \pm 2.76 in control vs. $7.53 \pm 2.28$ in ADM group, $\mathrm{p}=.379$; Table 1$)$. There is no difference in the types of TBI in both groups $(\mathrm{p}=.921$; Table 1$)$.

\section{Table 1}

Demographic information and type of trauma in control and AMD group,

\begin{tabular}{|c|c|c|c|}
\hline & $\begin{array}{l}\text { Control } \\
(n=192)\end{array}$ & $\begin{array}{ll}\text { ADM } & \text { group } \\
(n=195) & \end{array}$ & $p$ \\
\hline $\begin{array}{l}\text { Ages, } \\
\text { Mean } \pm S D\end{array}$ & $41.74 \pm 15.69$ & $43.83 \pm 15.13$ & .183 \\
\hline Sex, $n(\%)$ & & & .033 \\
\hline Male & 129 (67.19) & $150(76.92)$ & \\
\hline Female & $63(32.81)$ & $45(23.08)$ & \\
\hline $\begin{array}{l}\text { GCS scalea }, \\
\text { Mean } \pm S D\end{array}$ & $7.45 \pm 2.76$ & $7.53 \pm 2.28$ & .379 \\
\hline $\begin{array}{l}\text { Type of } \\
\text { Trauma, } n(\%)\end{array}$ & & & .921 \\
\hline Contusion & $32(16.67)$ & $33(16.92)$ & \\
\hline $\begin{array}{l}\text { Intracranial } \\
\text { hematoma }\end{array}$ & $11(5.73)$ & 9 (4.62) & \\
\hline $\begin{array}{c}\text { Subdural } \\
\text { hematoma }\end{array}$ & 143 (74.48) & 145 (74.36) & \\
\hline SAH & $6(3.13)$ & $8(4.10)$ & \\
\hline
\end{tabular}

SAH, subarachnoid hemorrhage; ADM, artificial dura materials; GCS, glasgow coma scale.

${ }^{a}$ GCS scales were obtained on initial examination.

\section{Postoperative complications}

Postoperative complications were closely monitored in all patients, including subcutaneous hematoma, seizure, intracranial infection, CSF leakage, and foreign body reaction. The results showed that patients in control group have more complications than patients in ADM group $13.02 \%$ patients in control group vs. $4.1 \%$ in ADM group had subcutaneous hematoma $(\mathrm{OR}=3.349,95 \% \mathrm{CI} 1.460-7.678, p=.004$ after adjustment), $12.5 \%$ patients in control group vs. $5.64 \%$ in ADM group had intracranial infection $(\mathrm{OR}=2.432,95 \%$ CI $1.144-5.170$, $p=.021$ after adjustment), $13.02 \%$ patients in control group vs. $5.13 \%$ in ADM group had CSF leakage (OR $=2.689,95 \%$ CI, $1.239-5.835$, $p=.012$ after adjustment), and $10.42 \%$ in control group vs. $3.08 \%$ in $\mathrm{ADM}$ group developed seizure $(\mathrm{OR}=3.705,95 \% \mathrm{CI}, 1.436-9.560$, $p=.007)$.

\section{Discussion}

\section{Goal of study}

In this large retrospective study, we aimed to evaluate the therapeutic effect of bovine-derived pericardium membrane as artificial dura material to repair dura defect of patients who had TBI.

\section{Summary of results}

One group of patients just receives standard decompressive craniectomy without dura repair (control group), and the other group receives dura repair after decompressive craniectomy (ADM group). The overall postoperative complications were compared in both groups, and the results showed that bovine-derived pericardium membrane has overall better clinical outcomes than control group, and bovine-derived pericardium membrane is good biomaterial for dura repair.

\section{Comparison with other studies}

Decompressive craniectomy has been widely used to treat patients with severe TBI. Usually, a part of skull and dura is removed in this procedure to reduce ICP, also open up cranial cavity to clear-out blood clots and dead brain tissues. Although this procedure significantly releases ICP, its beneficial effect was controversial and its efficacy in TBI was uncertain (Citerio \& Andrews, 2007; Ho, Honeybul, Lind, Gillett, \& Litton, 2011; Maas, Stocchetti, \& Bullock, 2008; Munch et al., 2000; Polin et al., 1997). Life-threaten complications can occur after decompressive craniectomy, especially for elderly patients (De Bonis et al., 2011), such as hydrocephalus (De Bonis, Pompucci, Mangiola, Rigante, \& Anile, 2010), interhemispheric subdural hygroma (De Bonis et al., 2013). However, decompressive craniectomy is not a risk factor for hemorrhagic contusions (Sturiale et al., 2012). A web-based prognostic model has been developed to predict the outcome of decompressive craniectomy and evaluate the benefits and complications of decompressive craniectomy (Honeybul \& Ho, 2014). However, there is a growing body of literature supporting the efficacy of decompressive craniectomy (Albanese et al., 2003; Mtaweh \& Bell, 2015; Wang et al., 2015), including reducing ICP (Dam Hieu, Sizun, Person, \& Besson, 1996), preventing brain edema (Burkert \& Paver, 1988; Burkert \& Plaumann, 1989; Guerra et al., 1999), and improving brain tissue oxygenation (Jaeger, Soehle, \& Meixensberger, 2003).

In addition to pericardium from different species, other tissues have been used to dura repair, such as porcine small intestinal submucosa (Bejjani \& Zabramski, 2007). However, there is no study to compare which species is superior to the others in BTI treatment. Potentially, xerography from other species may cause zoonotic diseases, and most commonly used products are bovine growth hormone and dura mater grafts, that potentially will have prion contamination. Prions represent a group of proteins with a unique capacity to fold into different conformations. Pathogenic prions have been shown to cause lethal neurodegenerative diseases in humans and animals. These diseases are sometimes infectious and hence referred to as transmissible spongiform encephalopathies (Norrby, 2011). In the past over, $60 \%$ reported prion diseases caused by cadaveric dura mater transplantation were from Japan, and it is related to frequent use of Lyodura (Bonda et al., 2016). After improvement of manufacture, the incidence is significantly reduced (Bonda et al., 2016). In this study, we used decellularized bovine pericardium membranes which meet criteria of Chinese FDA, and de-contamination of prion procedure has been performed. So far, no case of iatrogenic prion transmission has been reported with these products in China.

\section{Conclusion}

In summary, our study demonstrated that bovine-derived pericardium membranes are good artificial dural substitutes for decompressive craniectomy, and it associates less clinical complications than patients without dura repair.

\section{References}

1. Albanese J, Leone M, Alliez J. R, Kaya J. M., Antonini F., et al, (2003). Decompressive craniectomy for severe traumatic brain injury: Evaluation of the effects at one year. Critical Care Medicine, 31, $2535-2538$ 
10. Compagnone C, Murray G. D, Teasdale G. M, Maas A. I, Esposito D, et al, European Brain Injury Consortium . (2005). The management of patients with intradural post-traumatic mass lesions: A multicenter survey of current approaches to surgical management in 729 patients coordinated by the European Brain Injury Consortium. Neurosurgery, 57, 1183-1192. discussion 1183-1192

11. Dam Hieu P, Sizun J, Person H, \& Besson G. (1996). The place of decompressive surgery in the treatment of uncontrollable posttraumatic intracranial hypertension in children. Child's Nervous System: ChNS: Official Journal of the International Society for Pediatric Neurosurgery, 12, 270-275.

12. De Bonis P, Pompucci A, Mangiola A, Paternoster G, Festa R., et al, (2011). Decompressive craniectomy for elderly patients with traumatic brain injury: It's probably not worth the while. Journal of Neurotrauma, 28, 2043-2048.

13. De Bonis P, Pompucci A, Mangiola A, Rigante L, \& Anile C. (2010). Post-traumatic hydrocephalus after decompressive craniectomy: An underestimated risk factor. Journal of Neurotrauma, 27, 1965-1970.

14. De Bonis P, Sturiale C. L, Anile C, Gaudino S, Mangiola A, et al, (2013). Decompressive craniectomy, interhemispheric hygroma and hydrocephalus: A timeline of events? Clinical Neurology and Neurosurgery, 115, 1308-1312.

15. El Majdoub F, Löhr M, Maarouf M, Brunn A, Stenzel W., Ernestus R. I. (2009). Transmigration of fibrino-purulent inflammation and malignant cells into an artificial dura substitute (Neuro-Patch): Report of two cases. Acta Neurochirurgica, 151, 833-835. 